SHORT REPORT

\title{
High molecular weight caldesmon positive stromal cells in the capsule of hepatocellular carcinomas
}

\author{
H Nakayama, H Enzan, M Yamamoto, E Miyazaki, W Yasui
}

J Clin Pathol 2004;57:776-777. doi: 10.1136/jcp.2004.016279

Aims: To investigate the smooth muscle nature of the stromal cells in the capsule of hepatocellular carcinomas.

Methods: Immunohistochemical analysis using monoclonal antibody to high molecular weight caldesmon (HCD), a highly specific marker for smooth muscle cells, was performed in 33 encapsulated hepatocellular carcinomas and adjacent hepatic tissues.

Results: HCD positive stromal cells were detected in the capsule of 21 of the 33 hepatocellular carcinomas examined. Conclusions: The capsule of hepatocellular carcinomas contains smooth muscle cells.

C aldesmon is a major calmodulin binding protein that also interacts with actin filaments. ${ }^{1}$ Two molecular caldesmon (HCD) and low molecular weight caldesmonhave been purified. ${ }^{2}$ In particular, HCD, with a molecular weight $120-150 \mathrm{kDa}$, is associated with smooth muscle contraction and is localised exclusively to smooth muscle cells. ${ }^{2}$ In contrast to $\alpha$ smooth muscle actin, HCD is not detected in myofibroblasts, ${ }^{3}$ indicating that HCD could be used as a highly specific marker for smooth muscle cells and smooth muscle tumours. ${ }^{4}$ In the colorectum, pericryptal fibroblasts are positive for HCD. ${ }^{5}$

$\alpha$ Smooth muscle actin is an excellent marker for myofibroblasts and transformed hepatic stellate cells, ${ }^{6}$ but is not specific for smooth muscle cells. ${ }^{3}$ To investigate the presence of smooth muscle cells in hepatic stroma, immunostaining for HCD should be performed. There are no reports regarding immunohistochemical analysis for HCD positive stromal cells in liver neoplasms.

" $\alpha$ Smooth muscle actin is an excellent marker for myofibroblasts and transformed hepatic stellate cells, but is not specific for smooth muscle cells"

To investigate the smooth muscle nature of the stromal cells in the capsule of hepatocellular carcinomas, we examined the expression of HCD in the capsule of hepatocellular carcinomas and adjacent hepatic tissues.

\section{MATERIALS AND METHODS}

We examined 33 completely or partially encapsulated hepatocellular carcinomas and their adjacent hepatic tissues from 33 postmortem cases (eight patients with hepatitis $B$ virus (HBV) associated hepatic cirrhosis, 12 with hepatitis $\mathrm{C}$ virus (HCV) associated hepatic cirrhosis, and the remaining 13 with hepatic cirrhosis of unknown aetiology) in the department of molecular pathology, graduate school of biomedical sciences, Hiroshima University, Japan, from 1981 to 1999 . All of the 13 postmortem cases with unknown aetiology were before 1989, and laboratory examinations of HCV infection were not performed. Immunohistochemical studies were performed using a Histofine SAM-PO(multi) kit (Nichirei, Tokyo, Japan). A monoclonal antibody against HCD (clone h-CD; Dakopatts, Glostrup, Denmark; 1/50 dilution; microwave treatment) was used. Vascular smooth muscle cells served as positive controls for HCD. We regarded HCD positive cells in the capsule to be HCD positive stromal cells.

\section{RESULTS}

HCD positive stromal cells were detected in the capsule of 21 of the 33 hepatocellular carcinomas, whereas no HCD positive cells were found in the fibrous septa of the adjacent hepatic tissues examined (fig 1). Of the 21 hepatocellular carcinomas with HCD positive stromal cells, four were HBV associated lesions, nine were HCV associated lesions, and the remaining eight had an unknown aetiology.

\section{DISCUSSION}

The presence of intermediately differentiated smooth muscle cells in the capsule of hepatocellular carcinomas is suggested by immunohistochemical analysis using various smooth muscle markers (desmin, smooth muscle actin (clone 1A4, HHF35, CGA7), and smooth muscle myosin heavy chain (SM1, SM2)) and electron microscopy. ${ }^{7}$ Unfortunately, the antibodies used in the above mentioned study $^{7}$ are not specific for smooth muscle cells, but are also positive in myofibroblasts. ${ }^{3}$ Therefore, to confirm the presence of smooth

Abbreviations: $\mathrm{HBV} / \mathrm{HCV}$, hepatitis $\mathrm{B} / \mathrm{C}$ virus; $\mathrm{HCD}$, high molecular weight caldesmon

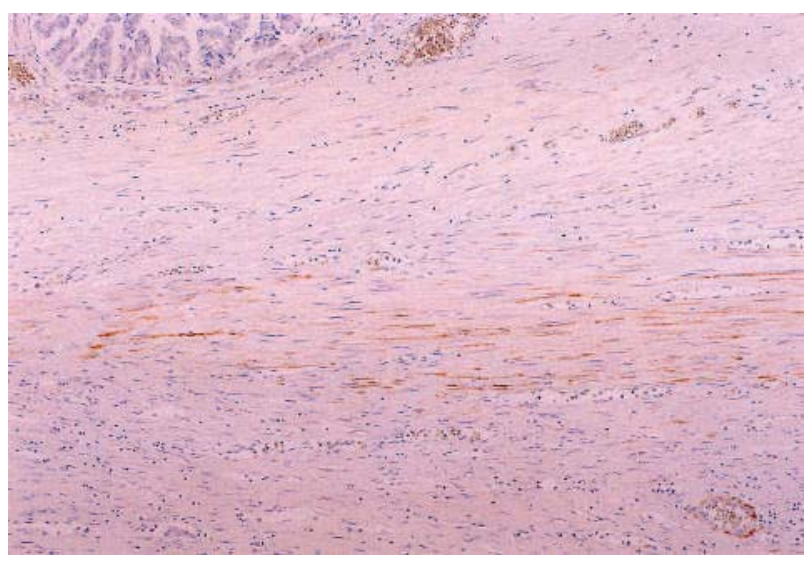

Figure 1 Immunostaining for high molecular weight caldesmon (HCD) in hepatocellular carcinoma. HCD positive stromal cells were detected in the fumour capsule; HCD was also positive in the vessel walls (vascular smooth muscle cells; lower right hand corner). 


\section{Take home messages}

- Immunohistochemical analysis using various smooth muscle markers has suggested that smooth muscle cells are present in the capsule of hepatocellular carcinomas, although the antibodies used were not specific

- We have confirmed this by the use of a monoclonal antibody to high molecular weight caldesmon, which is specific for smooth muscle cells

- Further molecular and biological analyses are needed to elucidate the origin of these cells

muscle cells, immunostaining for HCD should be performed. Recently, HCD positive stromal cells were detected in the capsule of thyroid follicular tumours and tumour-like lesions. ${ }^{8}$

In conclusion, HCD positive stromal cells, namely smooth muscle cells, were detected in the capsule of hepatocellular carcinomas. To elucidate the origin of the capsular smooth muscle cells, further molecular and biological investigations are needed.

\section{Authors' affiliations}

H Nakayama, W Yasui, Department of Molecular Pathology, Graduate School of Biomedical Sciences, Hiroshima University, 1-2-3 Kasumi Minami-ku, Hiroshima 734-8551, Japan
H Enzan, M Yamamoto, E Miyazaki, Department of Pathology, Kochi Medical School, Kohasu, Nankoku, Kochi 783-8505, Japan

Correspondence to: Dr H Nakayama, Department of Molecular Pathology, Graduate School of Biomedical Sciences, Hiroshima University, 1-2-3 Kasumi Minami-ku, Hiroshima 734-8551, Japan; nakayamh@hiroshima-u.ac.jp

Accepted for publication 9 February 2004

\section{REFERENCES}

1 Sobue K, Muramoto Y, Fujita M, et al. Purification of a calmodulin-binding protein from chicken gizzard that interacts with F-actin. Proc Natl Acad Sci U S A 1981;78:5652-6.

2 Ueki N, Sobue K, Kanda K, et al. Expression of high molecular weight caldesmon during phenotypic modulation of smooth muscle cells. Proc Natl Acad Sci U S A 1987;84:9049-53.

3 Lazard D, Sastre X, Frid MG, et al. Expression of smooth muscle-specific proteins in myoepithelium and stromal myofibroblasts of normal and malignant human breast tissue. Proc Natl Acad Sci U S A 1993:90:999-1003.

4 Miettinen MM, Sarlomo-Rikala M, Kovatich AJ, et al. Calponin and hcaldesmon in soft tissue tumors: consistent h-caldesmon immunoreactivity in gastrointestinal stromal tumors indicates traits of smooth muscle differentiation Mod Pathol 1999;12:756-62.

5 Nakayama H, Miyazaki E, Enzan H. Differential expression of high molecular weight caldesmon in colorectal pericryptal fibroblasts and tumor stroma. J Clin Pathol 1999;52:785-6.

6 Enzan H, Himeno $\mathrm{H}$, Iwamura S, et al. Alpha-smooth muscle actin-positive stromal perisinusoidal stromal cells in human hepatocellular carcinoma. Hepatology 1994; 19:895-903.

7 Kojima A, Kaneda K, Ueda M, et al. Induction of smooth muscle cells in the fibrous capsule of human hepatocellular carcinoma but not in the septa of hepatic cirrhosis. Virchow Arch 1999;434:413-22.

8 Nakayama H, Enzan H, Miyazaki E, et al. High molecular weight caldesmonpositive stromal cells in the capsule of thyroid follicular tumours and tumourlike lesions. J Clin Pathol 2002;55:917-20. 